\section{Case Reports in Ophthalmology}

\title{
Transmuscular Migration of a Scleral Tunnel-Secured Encircling Silicone Band
}

\author{
Yui Nishida Masanori Fukumoto Teruyo Kida Hiroyuki Suzuki \\ Tsunehiko Ikeda \\ Department of Ophthalmology, Osaka Medical College, Takatsuki City, Japan
}

\section{Keywords}

Transmuscular migration · Encircling silicone band · Scleral tunnel · Scleral buckling

\begin{abstract}
The migration of an encircling silicone band through a rectus muscle is a rare postoperative complication associated with scleral buckling surgery for retinal detachment. In this present study, we describe what we believe to be the first reported case of a patient who experienced postoperative migration of an encircling silicone band through the rectus muscle, despite the band being surgically secured to the sclera in a scleral tunnel. A 58-year-old man presented with a rhegmatogenous retinal detachment in his left eye. Pars plana vitrectomy was performed with the placement of an encircling silicone band, and the patient's retina was successfully reattached. One year postoperatively, the encircling band became exposed on the nasal side of the conjunctiva next to the limbus without any symptoms. Two weeks later, the exposed encircling band was surgically removed without any complications. The findings of this study show that even when an encircling silicone band is surgically secured around the eye, periodic and careful postoperative follow-up examinations should be performed to ensure no migration of the band.




\section{Case Reports in Ophthalmology}

Nishida et al.: Transmuscular Migration of a Scleral Tunnel-Secured Encircling Silicone Band

\section{Introduction}

The migration of an encircling silicone band through a rectus muscle is a rare postoperative complication associated with the scleral buckling procedure. However, both Kreis et al. [1] and Tan et al. [2] have reported cases of this rare complication. Here, we describe what we believe to be the first reported case of a scleral buckling-associated encircling silicone band that migrated through the rectus muscles, despite the band being securely surgically fixed to the sclera in the scleral tunnel; i.e., a review of the video recording of the surgery confirmed that the encircling band had been placed under the medial rectus muscles during the scleral buckling surgery.

\section{Case Report}

A 58-year-old man presented with a rhegmatogenous retinal detachment in his left eye. Pars plana vitrectomy with the placement of an encircling silicone band (\#240; MIRA, Inc., Waltham, Ma., USA) was performed on the patient, and his retina was successfully reattached. The solid silicone band was placed under the four rectus muscles and surgically fixed in a scleral tunnel at all four quadrants (fig. 1). The scleral tunnels were prepared by making two vertical incisions of one-half of the scleral thickness, and then performing a lamellar dissection with a surgical blade. The anterior extent of each tunnel was $2.0-2.5 \mathrm{~mm}$ posterior to the rectus muscle insertions.

One year later, the patient complained of a mild foreign body sensation in his left eye. Upon examination, the encircling band was found to be exposed on the nasal side of conjunctiva next to the limbus (fig. 2). The patient did not report any diplopia, and the movement of the associated eye in the field of the medial rectus muscle was found to be normal. An ophthalmoscopy examination revealed that his retina was still well attached. Two weeks later, the encircling silicone band was surgically removed without any complications. During that surgery, it was noted that the encircling band had cheese-wired through the medial rectus muscle, which had become reattached close to its original insertion with no thinning. At the patient's most recent follow-up, his retina remained well attached.

\section{Discussion}

Although it has been reported that a solid silicone band can cheese-wire forward through the rectus muscle postoperatively $[3,4]$, the underlying mechanism of that migration has yet to be elucidated. It has been suggested that a migration of an encircling band may occur if the band is embedded too deeply, placed anterior to the equator, and not securely anchored to the sclera [4]. In the case shown in this present study, the encircling band was fixed in the scleral tunnel at all four quadrants. A previous study has shown that as with the use of sutures, the use of a scleral tunnel is an alternative and secure method of anchoring an encircling band [5]. However, in the case shown in this present study, we theorize that the band migration possibly resulted from the scleral flaps being thin. An alternative explanation as to why the band migrated is that it might have been placed over the rectus muscle. However, a review of the video recording of the surgery confirmed that the encircling band had actually been placed beneath the muscle. 
It has been reported that migrating bands can induce ocular motility disturbances [3, 4]. Tan et al. [2] reported a case with ocular motility disturbances in whom reattachment of the cheese-wired lateral rectus muscle was not observed. In the case shown in this present study, the cheese-wired medial rectus muscle was found to have become reattached close to its original attachment site. We theorize that this was the reason why the ocular motility in our patient was preserved. Reportedly, the slow process of cheese-wiring migration through rectus muscle allows sufficient time for the muscle to reattach spontaneously behind the migrating band [4].

In conclusion, the findings of this study show that migration of an encircling element can occur within 1-year after placement, and with subtle symptoms such as foreign body sensation. To prevent cheese-wiring migration, a scleral tunnel that is both deep and long enough to securely fix the silicone band is recommended. Even when an encircling silicone band is surgically secured around the eye, periodic and detailed postoperative follow-up examinations and careful surgical procedures should be performed to ensure no migration of the band.

\section{Statement of Ethics}

The authors have no ethical conflicts to disclose.

\section{Disclosure Statement}

The authors have no conflicts of interest to disclose.

\section{References}

1 Kreis AJ, Klainguti G, Bovey EH, Wolfensberger TJ: Transmuscular migration of 240 silicone encircling band. Eye 2006;20:1456-1458.

-2 Tan LT, Thackare N, Zohdy G, Roberts-Harry J: Transmuscular migration of solid silicone band. Eye 2007;21:1013-1014.

- 3 Lanigan LP, Wilson-Holt N, Gregor ZJ: Migrating scleral explants. Eye 1992;6:317-321.

-4 Maguire AM, Zarbin MA, Eliott D: Migration of solid silicone encircling element through four rectus muscles. Ophthalmic Surg 1993;24:604-607.

5 Williams GA, Aaberg TM Jr: Techniques of Scleral Buckling; in Ryan SJ, Hinton DR, Schachat AP, Wilkinson CP. Retina, 4th ed. Mosby, St Louis, 2005, pp 2042-2050. 

www.karger.com/cop

Nishida et al.: Transmuscular Migration of a Scleral Tunnel-Secured Encircling Silicone Band

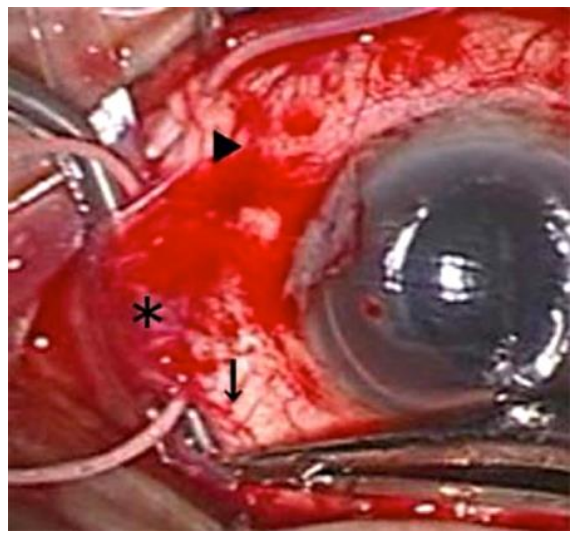

Fig. 1. A 240 silicone band is passed beneath the medial rectus muscle (asterisk) of the patient's left eye, and one of the ends (arrow) is then caught by the forceps. The other end of the band is secured to the sclera through the scleral tunnel (arrowhead).

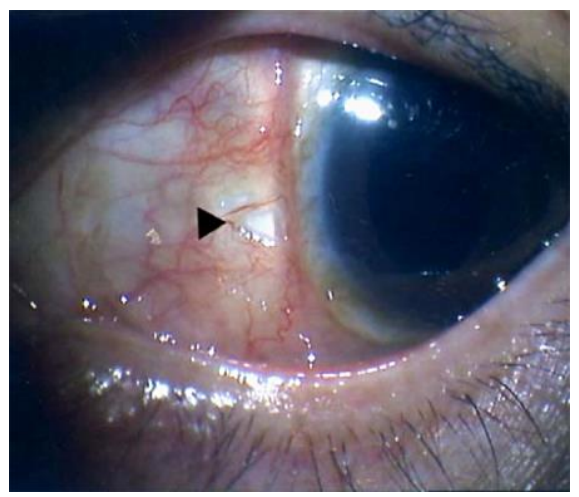

Fig. 2. The exposed 240 encircling silicone band through the conjunctiva in the patient's left eye at the nasal limbus (arrowhead). 